\title{
Effects of a Novel Formulation on Oral Biofilm, pH Buffering, and Gingival Health in Patients with Dry Mouth
}

\author{
Thair Takesh, Jessica Ho, Miracle Vania Firmalino, Delaney Islip, Afarin Anbarani, \\ and Petra Wilder-Smith
}

Beckman Laser Institute, University of California, Irvine, CA 92612, USA

Correspondence should be addressed to Petra Wilder-Smith; pwsmith@uci.edu

Received 16 February 2018; Revised 10 July 2018; Accepted 2 August 2018; Published 23 September 2018

Academic Editor: Carlos A. Munoz-Viveros

Copyright ( $\odot 2018$ Thair Takesh et al. This is an open access article distributed under the Creative Commons Attribution License, which permits unrestricted use, distribution, and reproduction in any medium, provided the original work is properly cited.

\begin{abstract}
Goal. To identify in patients with dry mouth the effects of a novel test agent (Oral Essentials Hydrating Formula Mouthwash, Beverly Hills, CA) versus a control agent (Biotène Dry Mouth Oral Rinse, GlaxoSmithKline Consumer Healthcare L.P., Moon Township, PA, USA) versus no treatment on dry mouth, plaque, salivary $\mathrm{pH}$ and buffering capacity, gingival health, and tooth sensitivity. Materials and Methods. In this cross-over study, ten subjects with dry mouth used test and control dry mouth interventions, as well as no dry mouth intervention in randomized sequence. Plaque Index, Gingival Index, Sulcus Bleeding Index, Plaque staining, and photographs were recorded at baseline and end of each study arm. Salivary volume, $\mathrm{pH}$, and buffering capacity were also recorded at these time points. Additionally, subjects completed a questionnaire for dry mouth and dentinal sensitivity at each visit. Results. Reductions in plaque presence and clinical indices were similar after use of test or control products $(p<0.05)$. Saliva volume and $\mathrm{pH}$ buffering improved significantly after use of test and control products $(p<0.05)$. Conclusions. The effects of a novel dry mouth intervention are similar to those of an existing OTC remedy and are significantly better than no intervention.
\end{abstract}

\section{Introduction}

With a reported prevalence of $5 \%$ to $46 \%$ [1], dry mouth has many negative sequellae $[2,3]$. As saliva lubricates and cleanses the mouth, protects teeth through its buffering and remineralizing properties, supports antimicrobial activity and hard tissue remineralization [4], and assists with chewing and speech, adequate salivation is essential to oral health and comfort. Inadequate saliva presence is more common in the elderly. One study reported persistent dry mouth in $17.5 \%$ of the elderly surveyed, with significantly higher prevalence in women [5]. With increasing age, chronic conditions that can cause dry mouth become more common, and the associated medications can further exacerbate the overall reduction in salivary presence, as well as variations in its biochemical composition and functions [6-15]. Other causes of dry mouth include head and neck radiotherapy [16], salivary gland disorders [17], diabetes [17], and Sjögren's syndrome [18].
Dry mouth symptoms range from mild discomfort to considerable oral disease that may impact oral health and even the patient's quality of life [3]. Oral and dental consequences include sensations of soreness and burning, difficulties in speaking, chewing and swallowing [17], mucosal atrophy [19], poor denture retention [20], and an altered oral microbiome [20-25]. Moreover, a significantly higher prevalence of enamel demineralization, dentinal sensitivity, and progressive dental caries, as well as periodontal disease is reported in patients with chronic dry mouth [20].

Treatment for dry mouth typically takes 2 forms: (1) addressing etiological factors and (2) alleviating symptoms and/or increasing salivary flow $[1,17]$. Systemic medications to stimulate saliva secretion include pilocarpine and cevimeline [26]. Moreover, a wide range of over-the-counter agents are available, including mouthwashes, rinses, sprays and gums, or salivary substitutes. Nevertheless, dry mouth remains an inadequately managed, common and chronic complaint, especially among the geriatric population [27]. 
The goal of this clinical study was to identify, in patients with dry mouth, the effects of (1) a novel test agent (Oral Essentials Hydrating Formula Mouthwash, Beverly Hills, CA) versus (2) a control agent that is commonly used by individuals with dry mouth (Biotène Dry Mouth Oral Rinse, GlaxoSmithKline Consumer Healthcare L.P., Moon Township, PA, USA), and (3) no treatment on plaque presence, salivary $\mathrm{pH}$ and buffering capacity, gingival health and tooth sensitivity, as well as intraoral comfort and function. These goals were formulated to test the hypothesis that the novel mouthwash has similar effects on these variables as one of the leading dry mouth agents, Biotène Dry Mouth Oral Rinse.

\section{Materials and Methods}

2.1. Human Subjects. This study was performed in full compliance with University of California IRB-approved protocol 2013-9778. All work was conducted in accordance with the Declaration of Helsinki (1964), with the human subjects' understanding and written consent. Ten subjects with confirmed dry mouth participated in this study. They were recruited at the University of California, Irvine by word-of-mouth, flyers, and e-mail recruitment. All subjects signed an informed consent at the beginning of the study, as well as statement of patient rights and photographic release forms. Subjects received an incentive payment at study completion.

\subsubsection{Subject Inclusion/Exclusion Criteria}

Inclusion criteria:

(1) Males and female subjects of all races and ethnicities, age between 18 and 75 years old

(2) Demonstrated an unstimulated whole saliva flow rate below $0.2 \mathrm{ml}$ per minute and a stimulated saliva flow rate less than $0.5 \mathrm{ml}$ in 5 minutes

(3) At least 5 natural teeth present in each quadrant (excluding third molars)

Exclusion criteria:

(1) Participation in any other clinical study involving the mouth or xerostomia within the last 30 days prior to enrolment into this study.

(2) Pregnant or nursing women (self-reported).

(3) Subjects who were unable to defer dental treatment during the study dates.

(4) History of significant adverse effects following use of oral hygiene products such as toothpastes and mouth rinses or allergy to personal care/consumer products or their ingredients.

(5) Significant past unresolved or current medical problem history.

(6) Presence of other major pathologies, such as herpetic infection, major recurrent apthous ulcers, or other ulcer forming diseases, abscesses, granulomas, or severe gingivitis, which might compromise the ability to perform measurements.
(7) Other significant disease or disorders that, in the investigator's opinion, would exclude the subject from the study including systemic conditions that would influence the course of periodontal disease.

(8) Active acquired immunodeficiency syndrome (AIDS) or hepatitis B/C (self-reported).

(9) Smokers.

(10) Self-reported GERD condition.

(11) Presence of any condition, abnormality or situation at baseline that in the opinion of the Principal Investigator night preclude the volunteer's ability to comply with study requirements, including completion of the study or the quality of the data.

Subject restrictions:

(12) Subjects would not be allowed to receive dental treatment (except emergency treatment) during the study.

(13) Subjects would be asked to refrain from all nonstudy oral hygiene procedures other than their usual brushing, flossing, and mouth washing routine during the study.

(14) Subjects embarking on a course of medication during the study would have to inform the Study Coordinator so that a decision could be made as to whether they could continue in the study. A fiveminute unstimulated saliva test might be taken to verify no change in saliva flow resulting from use of the new medication. Compliance with the protocol was checked at each test visit and recorded on the appropriate documentation.

2.2. Protocol. The study had 3 arms, whereby in two arms, subjects used a test or control dry mouth intervention; in the third arm, they used no intervention for xerostomia (Figure 1). Subjects were prerandomized with regard to interventional sequence using online randomizer software (Research Randomizer software: https://www.randomizer. org/). Test and control formulations were provided in numbered plain white containers to blind subjects and investigators with regard to treatment allocation. For the "no treatment" category, subjects were asked to refrain from any symptomatic dry mouth treatment; therefore, in this section of the study, the patient was not blinded. However, the clinical evaluator remained blinded as to the treatment allocation. Subjects were provided with a new standard Oral B ProFlex toothbrush (Procter \& Gamble, Cincinnati, $\mathrm{OH}, \mathrm{U}$. S.) for each arm of the study and instructed to continue with their usual oral hygiene measures.

After obtaining informed written consent during the baseline (Day 0) visit, standardized photographs as well as full-mouth Plaque Index (PI) [28], Gingival Index (GI) [29], and Sulcus Bleeding Index (mSBI) [30] were recorded for all teeth by an experienced clinician precalibrated to $95 \%$ consistency for all 3 indices in 100 periodontal patients over the past 6 months. Plaque was stained (2-Tone Disclosing Agent, Young Dental, Earth City, MO) and photographed at baseline and at the end of each study arm. Standardized photographs of the buccal/labial surfaces of all teeth were 


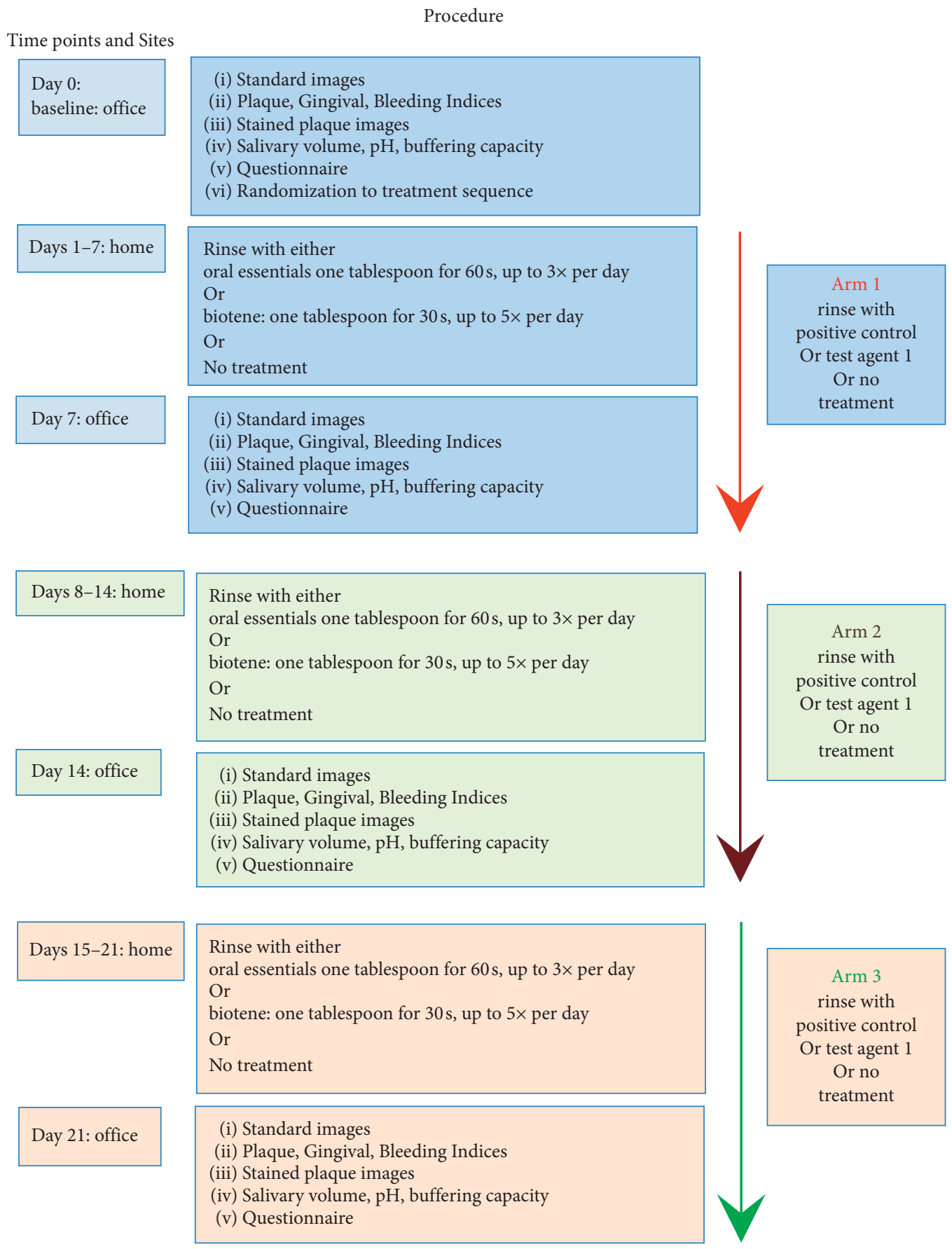

FIgURE 1: Flow chart of study protocol.

recorded by the same clinician using a Nikon D3200 camera with $18-55 \mathrm{~mm}$ lens and ring flash. A dental photographic mirror was used as necessary to visually access all surfaces. Salivary volume, $\mathrm{pH}$, and buffering capacity were also determined. Saliva was collected during office visits at baseline and at the end of each study arm by asking the seated subject to pool saliva in the floor of their mouth for 5 minutes, then to expectorate the saliva into a sterile graduated collecting cup. Subjects recorded daily how many times they used each product. Additionally, they completed a standardized selfevaluation questionnaire for dry mouth, dentinal sensitivity, and product evaluation at each visit (Figure 1).

\subsection{Products and Method of Use}

2.3.1. Test Product. Oral Essentials Hydrating Formula Mouthwash, Beverly Hills, CA, USA. Use approximately one tablespoon, swish vigorously for 60 seconds, and then spit out. Use up to 3 times per day.

2.3.2. Control Product. Biotène Dry Mouth Oral Rinse was obtained from GlaxoSmithKline Consumer Healthcare L.P., Moon Township, PA, USA. Use approximately one tablespoon, rinse for 30 seconds, and then spit out. Use up to 5 times per day. 
2.4. Data Analysis. Image J software (https://imagej.nih. gov/ij/) was used to process the digital intraoral photographs of the buccal/labial surface of each tooth, subject, and timepoint. This was achieved using the standard technique, whereby the borders of all plaque accumulations are visually delineated, and the areas thus mapped are expressed as percent coverage of each tooth surface. Digital image analysis also evaluated plaque age at each location, based on stain color (red stain indicates new plaque less than $24 \mathrm{~h}$ in age; blue stain indicates old plaque more than $24 \mathrm{~h}$ in age).

The value for each clinical index for each tooth unit at the end of each washout period was used as the baseline value for the subsequent arm of the study. The effects of each dentifrice on each clinical index and on plaque presence were tested using sums and differences of changes between study arms, calculated for each subject. Means and standard deviations were calculated for each group. The sums and differences were tested for significance by means of a twosample $t$-statistic. A two-sample $t$-test was also performed on the differences in the changes in each arm to see whether one treatment was more effective than the other.

\section{Results and Discussion}

3.1. Subject Questionnaire. All subjects completed a routine self-evaluation questionnaire for dentinal sensitivity, dry mouth status, and mouthwash ease of use at every visit (Figure 2).

3.1.1. Oral Health Questionnaire. Clinical criteria showed outstanding and highly significant $(p<0.01)$ improvement during the test and control agent use arms of the study except for "oral comfort at waking in the morning" (significant effect only for test intervention) and "do you sip liquids to aid swallowing" (no significant change from baseline for test and control interventions).

3.1.2. Agent Questionnaire. Overall, subject response was very positive. Ease of use, flavor, and mouth feel all scored highly at $>70 / 100$. Almost $80 \%$ of subjects stated that they would prefer a longer-lasting product for test and control products.

Overall, functions related to eating, sleeping, and overall oral comfort were improved using either intervention versus no intervention. This seems reasonable, as both the test and control formulations target symptomatic relief rather than long-term physiological functions. The level of symptomatic improvement is similar to that reported in other studies [28].

3.2. Plaque. Surface plaque was quantified three times: at baseline, and after each 7-day study arm, respectively (Figure 3). Plaque on oral surfaces was stained using a plaque-disclosing solution (2-Tone Disclosing Agent, Young Dental, Earth City, MO), and standardized intraoral photographs were recorded to document the extent of the plaque staining on all natural teeth. Using image J software, two assessments were made: (a) Plaque age based on stain color (blue vs red)

(b) Percent of total tooth surface covered by plaque

Using Image J software, \% of surface coverage was quantified for old $(>24 \mathrm{~h})$ and new $(<24 \mathrm{~h})$ plaque for each study arm.

There was a significant reduction in old and new plaque presence after use of test or control products for 1 week versus no product $(p<0.05)$. Old and new plaque levels were similar for the test and the control products $(p>0.1)$. Old plaque presence fell to approximately $50 \%$ of the baseline value, while new plaque presence was reduced to approximately $60 \%$ of the baseline level. Typically, greater saliva presence and flow are associated with better lubrication and self-cleansing of the mouth [31], which are both linked to reduced plaque presence as well as easier and more effective oral hygiene, so this result is not unexpected.

3.3. Clinical Indices. Clinical indices were quantified three times: at baseline, and after each 7-day study arm, respectively, using 3 standard numerical scales: Plaque Index (Quigley-Hein, Turesky Modification Plaque Index) (PI), Gingival Inflammation (Loe and Silness Gingival Index) (GI), and Gingival Bleeding (mSBI) (Figure 4).

Generally, clinical indices were similar for the test and the control products $(p>0.1)$. There was a significant reduction in clinical Plaque Index by approx. 60\% after use of test or control products for 1 week $(p<0.05)$. This parallels the reduction in clinical plaque presence that was quantified using photographs and image quantification software (see previous paragraph). The reduction in Gingival Index after 7 days of test or control product use almost reached a significant level ( $p=0.0563$ for test product, $p=0.0643$ for control product). A lower Gingival Index is generally seen as an indication of better gingival health. This finding may be related to the reduction in plaque levels, which are typically related to an improvement in gingival health [32]. Because the duration of each study leg was relatively short, it is not possible to determine whether longer-term usage of the control or test interventions would have resulted in further improvements in the Gingival Index. The Sulcus Bleeding Index (mSBI) did not change significantly during the course of this study. The discrepancy between the findings of the G.I. versus the mSBI have been observed in many studies [33]. The GI is based on two of the characteristic signs of inflammation-swelling (edema) and redness as well as bleeding. The mSBI is more strongly focused on bleeding and edema [33]. One study directly comparing data from the 2 indices in the same patient group determined that although gingival edema and change in color are often found together, bleeding can occur independently of edema [33]. These data underline the importance of more extensive and comprehensive studies that are carefully controlled over adequate periods of time to ensure maximum validity for the data obtained.

3.4. Saliva. Salivary volume, $\mathrm{pH}$, and buffering capacity were recorded at the beginning and end of each study arm. Buffering measurements were made using GC Saliva-Check Buffer Kit. 


\begin{tabular}{|c|c|c|c|c|}
\hline \multirow{2}{*}{ Oral health questionnaire } & \multicolumn{4}{|c|}{ Mean score on $100 \mathrm{~mm}$ VAS (o-best; 100-worst) } \\
\hline & Baseline & None & Control & Test \\
\hline I) Dryness/wetness upon waking in the morning (0-wettest; 100-driest) & 85 & 80 & 45 & 45 \\
\hline II) Mouth comfortable (0)/ uncomfortable (100) upon waking in the morning & 90 & 85 & 90 & 35 \\
\hline 2. I have trouble maintaining my weight because of swallowing problems (0-no; 100 -a lot) & 0 & 0 & 0 & 0 \\
\hline 3. I have trouble eating certain solid foods (hard to chew, crumbly, sticky)(0-no; 100 -a lot) & 75 & 80 & 40 & 35 \\
\hline 4. I have trouble drinking thin liquids (like water, tea, and Ensure $\left.{ }^{\oplus}\right)(0-$ no; 100 -a lot $)$ & 0 & 0 & 0 & 0 \\
\hline 5. Food gets stuck in my mouth ( 0 -no; 100 -a lot $)$ & 65 & 55 & 5 & 10 \\
\hline 6. Food gets stuck in my throat ( 0 -no; 100 -a lot $)$ & 40 & 20 & 5 & 5 \\
\hline 7. I choke or strangle on liquids (0-no; 100 -a lot) & 0 & 0 & 0 & 0 \\
\hline 8. I choke or strangle on solid foods ( 0 -no; 100 -a lot) & 0 & 0 & 0 & 0 \\
\hline 9. I have problems with dry mouth (0-no; 100 -a lot) & 100 & 100 & 50 & 60 \\
\hline 10. The amount of saliva in my mouth seems to be too little ( 0 -no; 100 -a lot $)$ & 100 & 100 & 60 & 50 \\
\hline 11. Problems with me dry mouth make chewing and swallowing difficult (0-no; $100-\mathrm{a}$ lot) & 70 & 60 & 65 & 40 \\
\hline 12. Problems with dry mouth affect my ability to sleep ( 0 -no; 100 -a lot) & 0 & 0 & 0 & 0 \\
\hline 13. Do you sip liquids to aid in swallowing dry foods? (0-no; 100 -a lot) & 50 & 50 & 50 & 50 \\
\hline 14. Problems with dry mouth affect my ability to talk ( 0 -no; 100 -a lot $)$ & 0 & 0 & 0 & 0 \\
\hline 15. Are any of your teeth sensitive to hot, cold, or spicy food or drinks (0-no; 100 -a lot) & 70 & 70 & 60 & 40 \\
\hline \multicolumn{5}{|l|}{ Agent questionnaire } \\
\hline 16. How long does the effect last while sleeping (in hours)? & $\mathrm{N} / \mathrm{a}$ & $\mathrm{N} / \mathrm{a}$ & 4 & 3 \\
\hline 17. How long does the flavor last while sleeping (in hours)? & $\mathrm{N} / \mathrm{a}$ & $\mathrm{N} / \mathrm{a}$ & 4 & 6 \\
\hline 18. How long does the effect last during the day (in minutes)? & $\mathrm{N} / \mathrm{a}$ & $\mathrm{N} / \mathrm{a}$ & 180 & 150 \\
\hline 19. Please rate the ease of use & $\mathrm{N} / \mathrm{a}$ & $\mathrm{N} / \mathrm{a}$ & 80 & 80 \\
\hline 20. Please rate the flavor $(0-100)$ & $\mathrm{N} / \mathrm{a}$ & $\mathrm{N} / \mathrm{a}$ & 80 & 70 \\
\hline 21. Please rate the mouth feel immediately after use $(0-100)$ & $\mathrm{N} / \mathrm{a}$ & $\mathrm{N} / \mathrm{a}$ & 70 & 80 \\
\hline 22. Please rate the convenience $(0-100)$ & $\mathrm{N} / \mathrm{a}$ & $\mathrm{N} / \mathrm{a}$ & 80 & 80 \\
\hline 23. What is your overall impression of the product? $(0-100)$ & $\mathrm{N} / \mathrm{a}$ & $\mathrm{N} / \mathrm{a}$ & 70 & 70 \\
\hline 24. Will you continue to use this product/recommend it to others? $Y / N$ & $\mathrm{~N} / \mathrm{a}$ & $\mathrm{N} / \mathrm{a}$ & $Y(60 \%)$ & $Y(60 \%)$ \\
\hline \multicolumn{5}{|l|}{ Question 1a: $0=$ dry; $10=$ very wet } \\
\hline \multicolumn{5}{|l|}{ Question 1b: $0=$ uncomfortable; $10=$ comfortable } \\
\hline \multicolumn{5}{|l|}{ Questions 2-4: $0=$ none; $10=\mathrm{a}$ lot } \\
\hline \multicolumn{5}{|l|}{ Questions 5-14: $0=$ never; $10=$ always } \\
\hline \multicolumn{5}{|l|}{ Question 15: $0=$ no sensitivity; $10=$ severe } \\
\hline \multicolumn{5}{|l|}{ Question 19: $0=$ very easy; $10=$ very difficult } \\
\hline \multicolumn{5}{|l|}{ Questions $20-22: 0=$ best; $10=$ worst } \\
\hline \multicolumn{5}{|l|}{ Question 23: $0=$ not good; $10=$ very good } \\
\hline Question 24: $0=$ no; $10=$ definitely & & & & \\
\hline
\end{tabular}

FIGURE 2: Questionnaire self-evaluation responses.

3.4.1. Saliva Volume. The volume of saliva collected over 5 minutes in subjects with xerostomia was approximately doubled at the end of the test and control study arms, representing a statistically significant increase in each case $(p<0.05)$ (Table 1$)$. In the study arm with no intervention, saliva production did not change significantly $(p>0.1)$. The transient increase in saliva production is similar to that reported in other studies using similar products $[7,34]$.

3.4.2. Salivary $p H$. $\mathrm{pH}$ values did not differ significantly between study arms and timepoints $(p<0.05)$ (Table 2).

3.4.3. Salivary Buffering Capacity. Salivary $\mathrm{pH}$ buffering performance improved significantly in test and control arms of the study $(p<0.05)$. No change was observed in the arm when no intervention was used $(p>0.1)$ (Table 3$)$.

Overall, this pilot study demonstrated that a novel dry mouth intervention has similar effects on plaque presence, salivary $\mathrm{pH}$ and buffering capacity, gingival health, and tooth sensitivity, as well as intraoral comfort and function as those of an existing OTC remedy [35-37]. The performance of the OTC remedy identified in this study resembled that documented in previous studies [35-37] and is significantly better than no intervention. Further studies are required in more patients and over a longer period of time.

\section{Conclusions}

In this first pilot study, the hypothesis was confirmed that a novel dry mouth intervention has similar effects as those of 


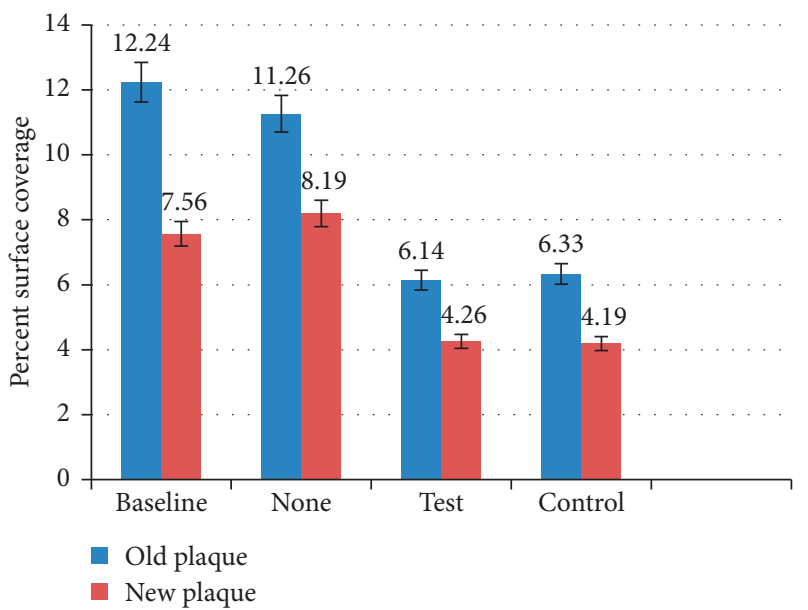

FIGURE 3: Mean (SD) plaque presence at baseline and after each 7-day arm of the study, expressed as \% of image surface area covered by old $(>24 \mathrm{~h})$ and new $(<24 \mathrm{~h})$ plaque, respectively.

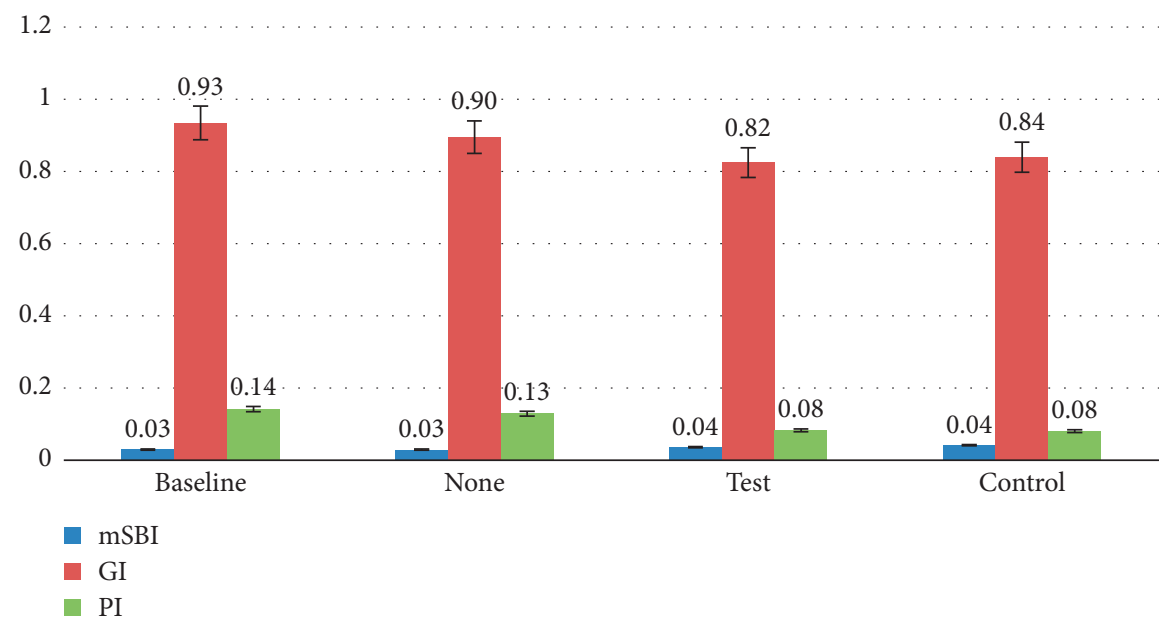

FIgURE 4: Mean clinical indices (SD) at baseline, and after each 7-day arm of the study.

TABLE 1: Mean saliva volume (SD) collected over 5 minutes in subjects at the beginning and end of each study arm.

\begin{tabular}{lccc}
\hline Baseline (ml (SD)) & No intervention $(\mathrm{ml}(\mathrm{SD}))$ & Test $(\mathrm{ml}(\mathrm{SD}))$ & Control (ml (SD)) \\
\hline $2.25 \mathrm{ml}(0.35)$ & $2.64 \mathrm{ml}(0.27)$ & $5.45 \mathrm{ml}(0.48)$ & $5.02 \mathrm{ml}(0.42)$ \\
\hline
\end{tabular}

TABLE 2: Mean salivary $\mathrm{pH}$ at baseline and at the end of each study arm.

\begin{tabular}{lccc}
\hline Baseline pH (SD) & No intervention pH (SD) & Test pH (SD) & Control pH (SD) \\
\hline $7.25(0.75)$ & $7.13(0.67)$ & $7.20(0.68)$ & $7.25(0.62)$ \\
\hline
\end{tabular}

TABLE 3: $\mathrm{pH}$ buffering capacity at (i) baseline and (ii) at the end of each study arm.

\begin{tabular}{ccccc}
\hline & Baseline & None & Test & Control \\
\hline pH buffering & Low (10/10 subjects) & Low $(10 / 10$ subjects $)$ & $\begin{array}{c}\text { Normal }(8 / 10 \text { subjects }) \\
\text { low }(2 / 10 \text { subjects })\end{array}$ & $\begin{array}{c}\text { Normal }(8 / 10 \text { subjects }) \\
\text { low }(2 / 10 \text { subjects })\end{array}$ \\
\hline
\end{tabular}


an existing OTC remedy and is significantly better than no intervention. Further studies are required in more patients and over a longer period of time.

\section{Data Availability}

The data generated from this study include identifiable personal health information. Thus, they cannot be released for ethical and privacy reasons. Moreover, the datasets used to support this study are currently under embargo, while the research findings are commercialized. Requests for data, 12 months after initial publication, will be considered upon request with permission of UCI IRB.

\section{Conflicts of Interest}

The authors have no conflicts of interest.

\section{Acknowledgments}

This research was supported by LAMMP NIH/NIBIB P41EB05890, the Arnold and Mabel Beckman Foundation, University of California SOM Seed Grant, and Oral Essentials Inc.

\section{References}

[1] A. Villa, C. L. Connell, and S. Abati, "Diagnosis and management of xerostomia and hyposalivation," Therapeutics and Clinical Risk Management, vol. 11, pp. 45-51, 2014.

[2] S. F. Cassolato and R. S. Turnbull, "Xerostomia: clinical aspects and treatment," Gerodontology, vol. 20, no. 2, pp. 64-77, 2003.

[3] J. M. Plemons, A. Al-Hashimi, and C. L. Marek, "Managing xerostomia and salivary gland hypofunction," Journal of the American Dental Association, vol. 145, no. 8, pp. 867-873, 2014.

[4] W. R. Thelin, M. T. Brennan, P. B. Lockhart et al., "The oral mucosa as a therapeutic target for xerostomia," Oral Diseases, vol. 14, no. 8, pp. 683-689, 2008.

[5] J. Y. An, R. Darveau, and M. Kaeberlein, "Oral health in geroscience: animal models and the aging oral cavity," Geroscience, vol. 40, no. 1, pp. 1-10, 2018.

[6] J. Ho, M. V. Firmalino, A. G. Anbarani, T. Takesh, J. Epstein, and P. Wilder-Smith, "Effects of a novel disc formulation on dry mouth symptoms and enamel remineralization in patients with hyposalivation: an in vivo study," Dentistry, vol. 7, no. 2, p. 411, 2017.

[7] M. S. Hopcraft and C. Tan, "Xerostomia: an update for clinicians," Australian Dental Journal, vol. 55, no. 3, pp. 238244, 2010.

[8] A. Villa, A. Polimeni, L. Strohmenger, D. Cicciù, E. Gherlone, and S. Abati, "Dental patients' self-reports of xerostomia and associated risk factors," Journal of the American Dental Association, vol. 142, no. 7, pp. 811-816, 2011.

[9] M. S. Ahmad, A. Bhayat, M. S. Zafar, and K. H. Al-Samadani, "The impact of hyposalivation on quality of life (QoL) and oral health in the aging population of Al Madinah Al Munawarrah," International Journal of Environmental Research and Public Health, vol. 14, no. 4, p. E445, 2017.

[10] S. C. Leal, J. Bittar, A. Portugal, D. P. Falcão, J. Faber, and P. Zanotta, "Medication in elderly people: its influence on salivary pattern, signs and symptoms of dry mouth," Gerodontology, vol. 27, no. 2, pp. 129-133, 2010.

[11] W. M. Thomson, "Dry mouth and older people," Australian Dental Journal, vol. 60, no. 1, pp. 54-63, 2015.

[12] B. Liu, M. R. Dion, M. M. Jurasic, G. Gibson, and J. A. Jones, "Xerostomia and salivary hypofunction in vulnerable elders: prevalence and etiology," Oral Surgery, Oral Medicine, Oral Pathology and Oral Radiology, vol. 114, no. 1, pp. 52-60, 2012.

[13] N. Kakudate, T. Muramatsu, M. Endoh et al., "Factors associated with dry mouth in dependent Japanese elderly," Gerodontology, vol. 31, no. 1, pp. 11-18, 2012.

[14] M. Alsakran Altamimi, "Update knowledge of dry moutha guideline for dentists," African Health Sciences, vol. 14, no. 3, pp. 736-742, 2014.

[15] H. Yousefi and M. Abdollahi, "An update on drug-induced oral reactions," Journal of Pharmacy and Pharmaceutical Sciences, vol. 21, no. 1, pp. 171-183, 2018.

[16] J. T. Johnson, G. A. Ferretti, W. J. Nethery et al., "Oral pilocarpine for post-irradiation xerostomia in patients with head and neck cancer," New England Journal of Medicine, vol. 329, no. 6, pp. 390-395, 1993.

[17] M. P. Lopez-Jornet, G. Garcia-Teresa, M. Vinas, and T. Vinuesa, "Clinical and antimicrobial evaluation of a mouthwash and toothpaste for xerostomia: a randomized, double-blind, crossover study," Journal of Dentistry, vol. 39, no. 11, pp. 757-763, 2011.

[18] R. Jonsson, U. Kroneld, K. Bckman, B. Magnusson, and A. Tarkowski, "Progression of sialadenitis in Sjögren's syndrome," Rheumatology, vol. 32, no. 7, pp. 578-581, 1993.

[19] A. Spielman, H. Ben-Aryeh, D. Gutman, R. Szargel, and E. Deutsh, "Xerostomia-diagnosis and treatment," Oral Surgery, Oral Medicine, Oral Pathology, vol. 51, no. 2, pp. 144-147, 1981.

[20] J. B. Epstein, S. Emerton, and P. Stevenson-Moore, "A doubleblind crossover trial of oral balance gel and Biotène toothpaste versus placebo in patients with xerostomia following radiation therapy," Oral Oncology, vol. 35, no. 2, pp. 132-137, 1999.

[21] M. Schiodt, L. B. Christensen, P. E. Petersen, and J. J. Thorn, "Periodontal disease in primary Sjögren's syndrome," Oral Diseases, vol. 7, no. 2, pp. 106-108, 2001.

[22] P. Tschoppe and H. Meyer-Lueckel, "Effects of regular and highly fluoridated toothpastes in combination with saliva substitutes on artificial enamel caries lesions differing in mineral content," Arch Oral Biol, vol. 57, no. 7, pp. 931-9, 2012.

[23] V. Kirstilä, M. Lenander-Lumikari, E. Söderling, and J. Tenouvo, "Effects of oral hygiene products containing lactoperoxidase, lysozyme and lactoferrin on the composition of whole saliva and on subjective oral symptoms in patients with xerostomia," Acta Odontologica Scandinavica, vol. 54, no. 6, pp. 391-397, 1996.

[24] V. Kirstilä, M. Lenander-Lumikari, and J. Tenouvo, "Effects of a lactoperoxidase-system-containing toothpaste on dental plaque and whole saliva in vivo," Acta Odontologica Scandinavica, vol. 52, no. 6, pp. 346-353, 1994.

[25] A. Lucchese, M. Portelli, M. Marcolina et al., "Effect of dental care on the oral health of Sjögren's syndrome patients," Journal of Biological Regulators and Homeostatic Agents, vol. 32, no. 2, pp. 37-43, 2018.

[26] S. R. Porter, C. Scully, and A. M. Hegarty, "An update of the etiology and management of xerostomia," Oral Surgery, Oral Medicine, Oral Pathology, Oral Radiology, and Endodontics, vol. 97, no. 1, pp. 28-46, 2004. 
[27] R. M. Nagler, "Salivary glands and the aging process: mechanistic aspects, health-status and medicinal-efficacy monitoring," Biogerontology, vol. 5, no. 4, pp. 223-233, 2004.

[28] S. Turesky, N. D. Gilmore, and I. Glickman, "Reduced plaque formation by the chloromethyl analogue of vitamin C," Journal of Periodontology, vol. 41, no. 41, pp. 41-43, 1970.

[29] H. Löe and J. Silness, "Periodontal disease in pregnancy," Acta Odontologica Scandinavica, vol. 21, no. 6, pp. 533-551, 1963.

[30] E. Newbrun, "Indices to measure gingival bleeding," Journal of Periodontology, vol. 67, no. 6, pp. 555-561, 1996.

[31] F. Dost and C. Farah, "Stimulating the discussion on saliva substitutes: a clinical perspective," Australian Dental Journal, vol. 58, no. 1, pp. 11-17, 2013.

[32] S. C. De David, T. G. Mário, G. C. De Freitas, K. Z. Kantorski, U. M. E. Wikesjö, and C. H. C. Moreira, "Correlation between plaque control and gingival health using short and extended oral hygiene intervals," Clinical Oral Investigations, vol. 22, no. 7, pp. 2593-2597, 2018.

[33] L. Benamghar, J. Penaud, P. Kaminsky, F. Abt, and J. Martin, "Comparison of gingival index and sulcus bleeding index as indicators of periodontal status," Bulletin of the World Health Organization, vol. 60, no. 1, pp. 147-151, 1982.

[34] S. Duong, J. Youssef, P. Pimenta et al., "An imaging-based approach to the evaluation of xerostomia," Lasers in Surgery and Medicine, vol. 44, no. 6, pp. 482-489, 2012

[35] A. M. Kielbassa, S. P. Shohadai, and J. Schulte-Mönting, "Effect of saliva substitutes on mineral content of demineralized and sound dental enamel," Support Care in Cancer, vol. 9, no. 1, pp. 40-47, 2001.

[36] A. M. Kielbassa and H. Meyer-Lueckel, "Effects of saliva substitutes and mouthwash solutions on dentin," Schweiz Monatsschr Zahnmed, vol. 111, no. 9, pp. 1060-1066, 2001.

[37] H. Meyer-Lueckel, J. Schulte-Mönting, and A. M. Kielbassa, "The effect of commercially available saliva substitutes on predemineralized bovine dentin in vitro," Oral Diseases, vol. 8, no. 4, pp. 192-198, 2002. 


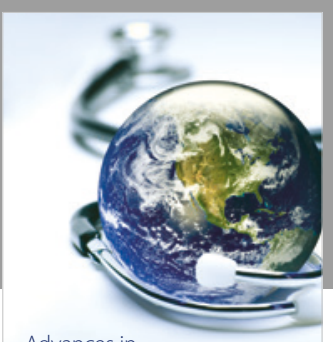

Advances in
Public Health

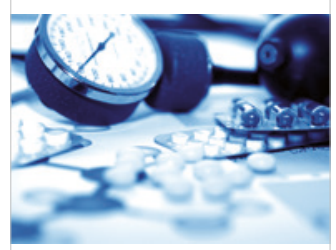

Case Reports in

Medicine

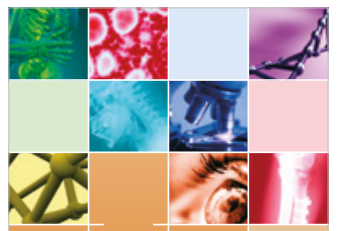

niernational Journal of

Biomaterials
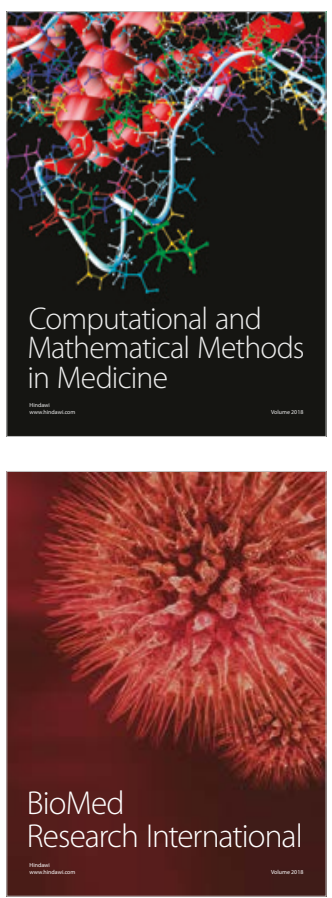

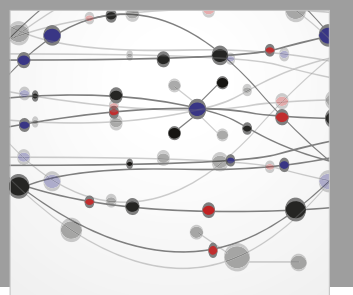

The Scientific World Journal Dentistry

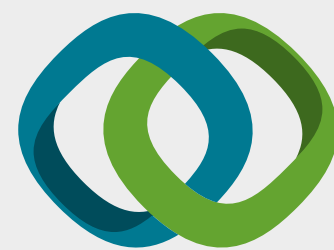

Hindawi

Submit your manuscripts at

www.hindawi.com
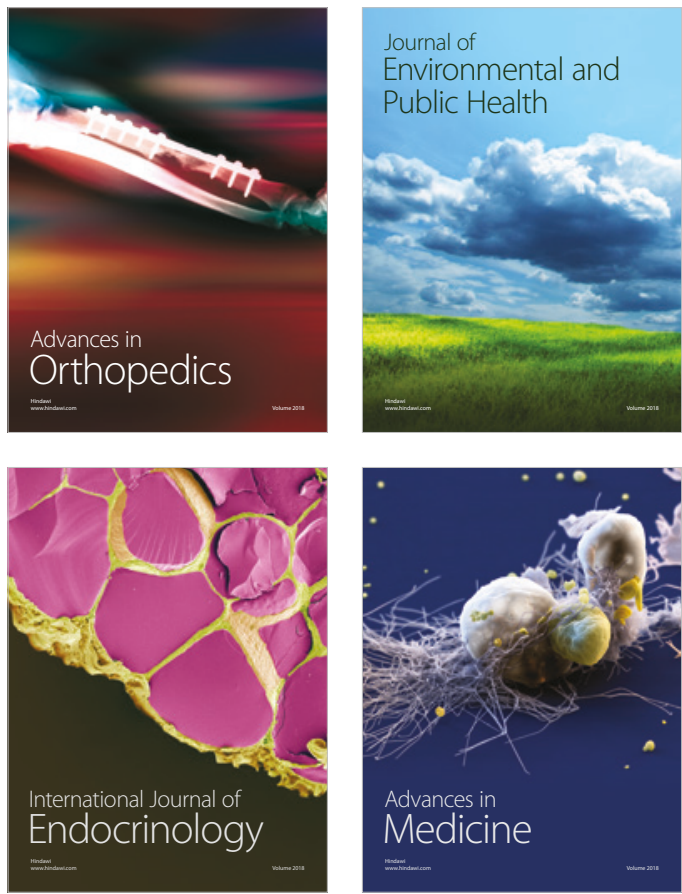
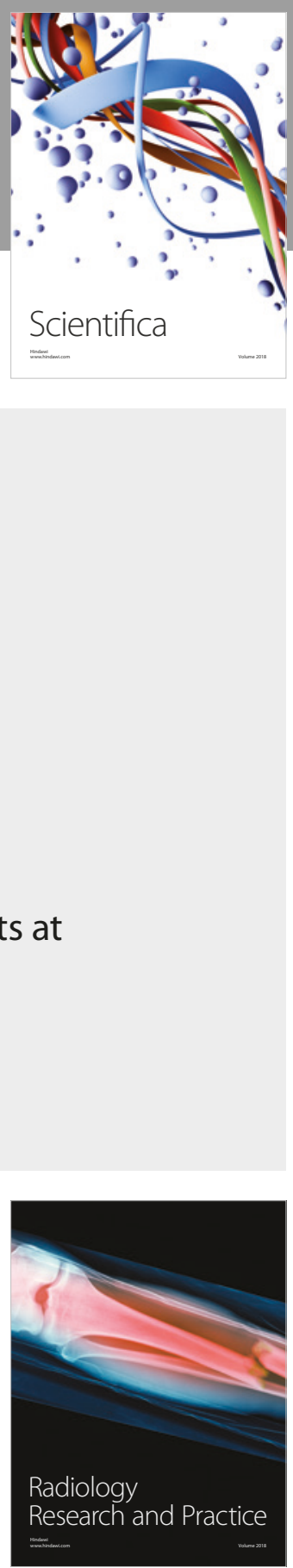

Scientifica

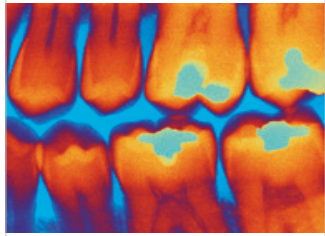

Case Reports in

Dentistry
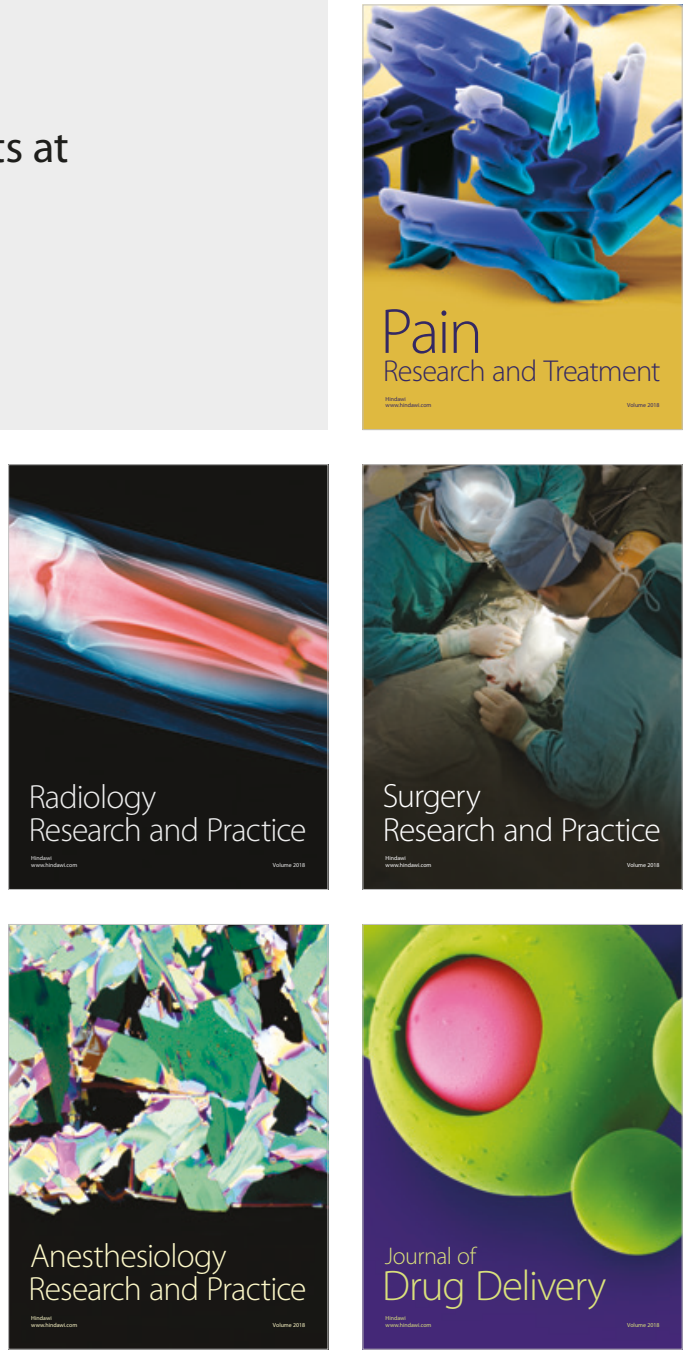